\title{
Precise CCD positions of Triton in 2014-2016 from the Gaia DR1 $\dagger$
}

\author{
N. Wang ${ }^{1}$, Q. Y. Peng ${ }^{1}$, H. W. Peng ${ }^{1,2}$ and Q. F. Zhang ${ }^{1}$ \\ ${ }^{1}$ Department of Computer Science, Jinan University,Guangzhou 510632, China, \\ email: tpengqy@jnu.edu.cn \\ ${ }^{2}$ Yunnan Observatory, CAS, Kunming 650216, China
}

\begin{abstract}
CCD observations during the years 2014-2016 have been reduced to derive the precise positions of Triton, the first satellite of Neptune. The observations were made by the 1 m telescope at Yunnan Observatory over 15 nights during the years 2014-2016. The theoretical position of Triton was retrieved from the Jet Propulsion Laboratory Horizons system. Our results show that when the newest Gaia catalogue (Gaia DR1) is referred to the mean O-Cs (observed minus computed) residuals are about 0.042 and -0.006 arcsec, the dispersions are 0.012 and 0.012 arcsec in right ascension and declination, respectively. The dispersions are improved very significantly when the Gaia DR1 is referred to. However, the agreement in right ascension is not so good as that in declination, the reason might come from the uncertainty of planet ephemeris. More observations are needed to confirm this.
\end{abstract}

Keywords. methods: observational, techniques: image processing, astrometry.

\section{Introduction}

Triton is the largest moon of Neptune and it has a retrograde, inclined and circular orbit. This unusual configuration has led to the belief that Triton originally orbited the Sun before being captured in orbit around Neptune (McKin 1984). Therefore Triton holds important clues to the evolution of the solar system. In recent years, generous observations of Triton have been made (Stone 2000; Vieira Martins et al. 2004; Qiao et al. 2014) and the precision of these observations is usually about 0.05 to 0.5 arcsec (Emelyanov \& Samorodov 2015). As the newest Gaia DR1 star catalogue is available (Gaia Collaboration 2016a,b), the precision of Triton must be further improved and a quite great CCD field of view allows us to calibrate its geometry accurately (Peng et al. 2012). Furthermore, the high precision of Triton can help improve the precision of Neptune (Robert et al. 2011).

\section{Observations}

Since 2014, we have been engaged in a systematic observation of Triton. All the observations were made with the $1 \mathrm{~m}$ telescope at Yunnan Observatory. The IAU site code is 286. A total of 755 frames of CCD images have been obtained for the satellite as well as 398 frames of CCD calibration images. The exposure time for each CCD frame ranged from 30 to $120 \mathrm{~s}$, depending on the meteorological conditions.

$\dagger$ This research work is supported by the National Science Foundation of China (Grant Nos U1431227, 11273014). This work has made use of data from the European Space Agency (ESA) mission Gaia (https://www.cosmos.esa.int/gaia), processed by the Gaia Data Processing and Analysis Consortium (DPAC, https://www.cosmos.esa.int/web/gaia/dpac/consortium). Funding for the DPAC has been provided by national institutions, in particular the institutions participating in the Gaia Multilateral Agreement. 

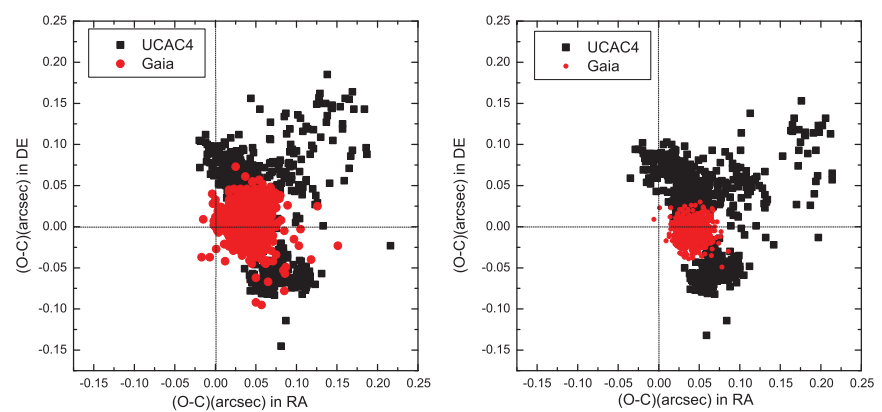

Figure 1. (O-C) residuals of Triton before and after Geometric Distortion Correction (GDC). The left panel shows the (O-C) residuals before GDC and the right panel after GDC.

Table 1. Statistics of (O-C) residuals for Triton by using different planetary and satellite ephemerides. Column 1 and 2 shows the planetary ephemeris and the satellite ephemerides respectively. The following columns list the mean (O-C) and its standard deviation (SD). Emelyanov15 means Emelyanov \& Samorodov (2015). All units are arcsec.

\begin{tabular}{cccccc}
\hline $\begin{array}{c}\text { planetary } \\
\text { ephemeris }\end{array}$ & $\begin{array}{c}\text { satellite } \\
\text { ephemeris }\end{array}$ & $\begin{array}{c}<\mathrm{O}-\mathrm{C}> \\
\text { RA }\end{array}$ & $\begin{array}{c}\text { SD } \\
<\mathrm{O}-\mathrm{C}> \\
\text { DEC }\end{array}$ & $\mathrm{SD}$ \\
\hline INPOP10 & Emelyanov15 & 0.083 & 0.013 & -0.099 & 0.013 \\
INPOP13C & Emelyanov15 & 0.062 & 0.013 & -0.059 & 0.013 \\
EPM2015 & Emelyanov15 & 0.062 & 0.013 & -0.014 & 0.013 \\
DE405 & Emelyanov15 & 0.149 & 0.016 & -0.028 & 0.014 \\
DE421 & Emelyanov15 & 0.057 & 0.013 & -0.018 & 0.013 \\
DE431 & Emelyanov15 & 0.042 & 0.013 & -0.007 & 0.013 \\
DE431 & JPL nep081xl & 0.042 & 0.012 & -0.006 & 0.012 \\
\hline
\end{tabular}

\section{Results and conclusions}

In order to analysis the observations, we compared our results using different referenced star catalogues, using different planetary ephemerides and satellite ephemerides.

The positional precision ( $\sim 12$ mas in each direction) of Triton has been greatly improved mainly due to the application of Gaia DR1 catalogue. The geometric distortion correction of a CCD frame has significant effect to the improvement of the positional precision of Triton. The ephemeris of Neptune even the new DE431 perhaps have a somewhat space ( $\sim 40$ mas in RA) to improve according to our present observations. On the other hand, the ephemerides of Triton from JPL and IMCCE have a very good agreement.

\section{References}

Emelyanov, N. V. \& Samorodov, M. Y. 2015, MNRAS, 454, 2205

Gaia Collaboration, Prusti, T., de Bruijne, J. H. J., et al. 2016, aap, 595, A1

Gaia Collaboration, Brown, A. G. A., Vallenari, A., et al. 2016, aap, 595, A2

McKinnon, W. B. 1984, nat, 311, 355

Peng, Q. Y., Vienne, A., Zhang, Q. F., et al. 2012, AJ, 144, 170

Qiao, R. C., Zhang, H. Y., Dourneau, G., et al. 2014, MNRAS, 440, 3749

Robert, V., de Cuyper, J.-P., Arlot, J.-E., et al. 2011, MNRAS, 415, 701

Stone, R. C. 2000, AJ, 120, 2124

Vieira, M. R., Veiga, C. H., Bourget, P., et al. 2004, aap, 425, 1107 